\title{
Recent Trends on Order Bounded Disjointness Preserving Operators ${ }^{1}$
}

\author{
KARIM BOULABIAR
}

\section{INTRODUCTION}

Disjointness preserving operators have been introduced in some form or other in the forty's. Indeed, linear multiplicative operations in the work [55] by Vulikh are disjointness preserving operators in disguise. However, only during the last decades have they made a formal entry into the development of vector lattices. A first systematic study of disjointness preserving operators goes back to the pioneering note [4] by Abramovich, Veksler, and Koldunov published in the end of the seventies. From then on, the interest in disjointness preserving operators has steadily grown and a series of works devoted to the subject appeared in the literature. In this regard, spectral properties of order bounded disjointness preserving operators were considered in great details in $[8,30]$. On the other hand, invertible disjointness preserving operators occupied a prominent role in a vast literature, such as $[7,20,33,35]$ and mainly the remarkable memoir [3] by Abramovich and Kitover. One of the external reasons for the continuing interest in disjointness preserving operators is the fact that precisely the order bounded disjointness preserving operators allow multiplicative representations as weighted composition operators and, more generally, polar decompositions $[2,19,28,39]$. They thus found applications in the theory of singular and integral equation, dynamical system, and differential equations with delayed time [38, 46, 51].

The present survey on order bounded disjointness preserving operators has two main objectives. First, convince the young researchers in vector lattices that disjointness preserving operators constitute an honorable research activity. Secondly, inform the experts about

\footnotetext{
${ }^{1}$ Most of the content of this survey was presented by the author in the Instructional Workshop organized by Professor Anthony Wickstead in the summer of 2008 at Queen's University Belfast.
} 
results on the subject obtained recently by the author. In fact, this is a study of modest length - selectivity is a must - with the choices of illustrations being left, for good or ill, to personal taste and prejudices. In this prospect, no attention has been paid to aspects of order bounded disjointness preserving operators evoked in the preceding paragraph, except of their representations as weighted composition operators.

The exposition is divided in six sections. This introduction is followed by a section dealing with what is considered today as the most important result in the theory of order bounded disjointness preserving operators, namely, Meyer's Theorem [43]. In the third section, order bounded disjointness preserving operators on certain spaces of continuous functions are characterized. In this prospect, some results are presumably known. However, the author has not been able to locate precise references for them. For this reason, complete proofs will be given. The fourth section contains extensions of results in Section 3 to the more general setting of functions algebra in the sense of Birkhoff and Pierce [15]. Section 5 gives a look at disjointness preserving operators from a different 'global' point of view. Indeed, the lattice structure of certain sets of order bounded operators preserving disjointness are investigated and facts on the socalled orthomorphisms are extended to such sets. Some results in this section are new and they will be proved completely. The last section concerns algebraic (in the sense of Kaplansky [37]) order bounded disjointness preserving operators. To be a little more precise, we focus on order bounded disjointness preserving operators that satisfy a nontrivial polynomial equation. At last, we point out that this survey contains some open problems. The hope of the author is that the reader will find them rather worthy of interest.

The books $[1,6,41,45,59]$ on the theory of vector lattices and operators between them are used in this survey as the sources of unexplained terminology and notation.

\section{MEYER's THEOREM}

Unless otherwise stated, $L$ and $M$ stand throughout for Archimedean vector lattices. A (linear) operator $T$ from $L$ into $M$ is said to be disjointness preserving (or to preserve disjointness) if

$f, g \in L$ and $|f| \wedge|g|=0$ in $L$ imply $|T(f)| \wedge|T(g)|=0$ in $M$. 
It is not hard to see that the operator $T$ from $L$ into $M$ preserves disjointness if and only if $|T(f)| \wedge|T(g)|=0$ for all $f, g \in L$ with $f \wedge g=0$. Also, $|T(f)|=|T(|f|)|$ for all $f \in L$ is a necessary and sufficient condition for the operator $T$ from $L$ into $M$ in order to be disjointness preserving. Obviously, the operator $T$ from $L$ into $M$ is a lattice homomorphism if and only if $T$ is a positive disjointness preserving operator. As mentioned in the title, this survey will deal with disjointness preserving operators that are order bounded. It should be pointed out here that a disjointness preserving operator need not be order bounded. An example in this direction is given next.

Example 2.1. A real-valued function $f$ defined on the real interval $[0, \infty)$ is said to be essentially polynomial if there exist a real polynomial $p_{f}$ and a real number $\lambda_{f}$ with $f(x)=p_{f}(x)$ for all $x \in\left[\lambda_{f}, \infty\right)$. The set $L$ of all essentially polynomial functions on $[0, \infty)$ is an Archimedean vector lattice with respect to the usual pointwise operations and ordering. The operator $T$ from $L$ into the vector lattice $\mathbb{R}$ of all real numbers defined by $T f=p_{f}(0)$ for all $f \in L$ preserves disjointness but is not order bounded.

At this point, recall that the set $\mathcal{L}(L, M)$ of all operators from $L$ into $M$ is an Archimedean ordered vector space with respect to the usual pointwise operation and ordering. The set $\mathcal{L}_{b}(L, M)$ of all order bounded operators from $L$ into $M$ is an ordered vector subspace of $\mathcal{L}(L, M)$. It is well-known that $\mathcal{L}(L, M)$ is not a vector lattice, in general. In this regard, even an order bounded operator from $L$ into $M$ need not have an absolute value nor in $\mathcal{L}(L, M)$ neither in $\mathcal{L}_{b}(L, M)$. In spite of that, $\mathcal{L}_{b}(L, M)$ is a Dedekind complete latticesubspace [1] of $\mathcal{L}(L, M)$ as soon as $M$ in addition Dedekind complete. This leads us to the what is considered today as the fundamental theorem of order bounded disjointness preserving operators, namely, the following very famous Meyer's Theorem.

Theorem 2.2. Let $T$ be an order bounded disjointness preserving operator from $L$ into $M$. Then there exist unique lattice homomorphisms $T^{+}, T^{-}$from $L$ into $M$ such that $T=T^{+}-T^{-}$and

$$
T^{+}(f)=(T(f))^{+}, T^{-}(f)=(T(f))^{-} \quad \text { for all } f \in L^{+} .
$$

In particular, $T$ has an absolute value $|T|$ in $\mathcal{L}(L, M)$ and $|T|=$ $T^{+}+T^{-}$. Moreover, $|T|$ is a lattice homomorphism from $L$ into $M$ 
such that

$$
|T|(|f|)=|T(f)|=|| T|(f)| \quad \text { for all } f \in L .
$$

The first proof of the Theorem 2.2 was given in [43] by Meyer himself. Meyer's proof is not constructive, that is, it is based upon the Zorn's Lemma (i.e., the Axiom of Choice). Later, two Zorn's Lemma free proofs of Theorem 2.2 were provided by Bernau in [12] and de Pagter in [47], respectively.

Meyer's Theorem is in many ways the starting point of our investigation of order bounded disjointness preserving operators. Let us collect some consequences. First of all, it is readily verified that the kernel $\operatorname{ker} T$ of a lattice homomorphism $T$ from $L$ into $M$ is an order ideal of $L$. Theorem 2.2 yields directly that if $T$ is an order bounded disjointness preserving operator from $L$ into $M$ then $\operatorname{ker} T=\operatorname{ker}|T|$. So, the kernel of any order bounded disjointness preserving linear operator is again an order ideal. However, contrary to lattice homomorphisms, the range $\operatorname{Im} T$ of the order bounded disjointness preserving linear operator $T$ from $L$ into $M$ need not be a vector sublattice of $M$. To see this, consider the order bounded disjointness preserving operator from the Archimedean vector lattice $C([0,1])$ of all real-valued continuous functions on the real interval $[0,1]$ into itself defined by $(T(f))(x)=x f(x)$ for all $f \in C([0,1])$ and $x \in[0,1]$. Another nice application of Meyer's Theorem was obtained by Huijsmans and Wickstead in [34]. That is, if $T$ is a bijective order bounded disjointness preserving operator from $L$ into $M$, then the inverse $T^{-1}$ is an order bounded disjointness preserving operator from $M$ into $L$. Moreover, the equality $|T|^{-1}=\left|T^{-1}\right|$ holds in $\mathcal{L}(M, L)$. In [8], Arendt proved that if $L$ and $M$ are Banach lattices then an operator $T$ from $L$ into $M$ is an order bounded disjointness preserving operator if and only if $|T(f)| \leq|T(g)|$ holds in $M$ whenever $|f| \leq|g|$ holds in $L$. Relying on Meyer's Theorem, [33] Huijsmans and de Pagter extended the characterization obtained by Arendt to arbitrary Archimedean vector lattices. This result is interesting in part because it gives one equivalent condition to both order boundedness and disjointness preservation. Since we are evoking Arendt and his work [8], we point out that he called order bounded disjointness preserving operators shortly Lamperti operators.

Now, we shift our emphasis from the general case to the particular setting of band preserving operators. Let $T$ be an operator on $L$ (i.e., from $L$ into $L$ ) and recall that a nonvoid subset $D$ of $L$ is 
said to be $T$-invariant whenever $T$ maps $D$ into $D$. An operator $T$ on $L$ is said to be band preserving if every band of $L$ is $T$-invariant. Hence, the operator $T$ on $L$ is band preserving if and only if $|T(f)| \wedge$ $|g|=0$ for all $f, g \in L$ with $|f| \wedge|g|=0$. Obviously, if any band preserving operator on $L$ preserves disjointness. This implication is not reversible, of course. On the other hand, the following example (obtained by Meyer in [44]) shows that a band preserving operators need not be order bounded.

Example 2.3. The set $P L([0,1))$ of all piecewise linear functions on $[0,1)$ is an Archimedean vector lattice with respect to the usual pointwise operations and ordering. Notice here that $f \in P L([0,1))$ if and only if there exists a partition $0=x_{0}<x_{1}<\ldots<x_{n-1}<x_{n}=$ 1 of $[0,1)$ such that $f$ is linear on $\left[x_{i-1}, x_{i}\right)$ for each $i \in\{1, \ldots, n\}$. The band preserving operator $T$ on $P L([0,1))$ defined by

$$
T(f)(x)=f_{r}^{\prime}(x) \quad \text { for all } f \in P L([0,1)) \text { and } x \in[0,1),
$$

where $f_{r}^{\prime}$ indicates the right derivative of $f$, is not order bounded.

Only order bounded band preserving operators will be considered in this study. In this prospect, an order bounded band preserving operator $T$ on $L$ is called an orthomorphism on $L$. Obviously, the identity operator $I_{L}$ on $L$ is an orthomorphism on $L$. Moreover, the set $\operatorname{Orth}(L)$ of all orthomorphisms on $L$ is an ordered vector subspace of $\mathcal{L}(L)=\mathcal{L}(L, L)$. Actually, Orth $(L)$ is much more than a simple ordered vector subspace of $\mathcal{L}(L)$. Indeed, Bigard and Keimel in [14] and, independently, Conrad and Diem in [24] proved that $\operatorname{Orth}(L)$ is a generalized vector sublattice (in the sense of [5]) of $\mathcal{L}(L)$ with the lattice operations given pointwise, meaning that, if $S, T \in \operatorname{Orth}(L)$ then $(S \vee T)(f)=S(f) \vee T(f)$ and $(S \wedge T)(f)=$ $S(f) \wedge T(f)$ for all $f \in L^{+}$. In particular, if $T \in \operatorname{Orth}(L)$ then the absolute value $|T|$ exists and $|T|(|f|)=|T(f)|=|| T|(f)|$ for all $f \in L$. The latter can be obtained alternatively from Theorem 2.2 since $T$ in particular preserves disjointness.

\section{Concrete Situations}

By and large, the notation and terminology of the great text [27] by Gillman and Jerison will be used in this section unless it conflicts with the by now standard notation used by workers in vector lattices. In particular, $\mathbb{R}^{X}$ will indicate the universally complete [6] vector lattice of all real-valued functions on a nonvoid set $X$ under the usual 
pointwise addition, scalar multiplication, and ordering. Moreover, the constant function on $X$ whose constant value is the real number $r$ is denoted by $\mathbf{r}_{X}$. Furthermore, if $X$ is a topological space then $C(X)$ denoted the (relatively) uniformly complete [41] vector sublattice of $\mathbb{R}^{X}$ of all continuous functions on $X$. The main objective of this section is to characterize order bounded disjointness preserving operators on $C(X)$-type vector lattices under suitable restrictions on $X$.

A topological space is called a Tychonoff space if it is a subspace of a compact Hausdorff space. In [54], Tychonoff himself proved that the topological space $X$ is Tychonoff if and only if $X$ is Hausdorff and completely regular, that is, whenever $F$ is a closed set in $X$ and $x_{F} \in X$ with $x_{F} \notin F$, there exists $f \in C(X)$ such that $f\left(x_{F}\right)=0$ and $f(x)=1$ for all $x \in F$. It was known to both Stone [53] and Cech [25] that for each topological space $X$, there is a Tychonoff space $X^{*}$ such that $C(X)$ and $C\left(X^{*}\right)$ are isomorphic as vector lattices. In fact, $X^{*}$ is obtained by first identifying those points which cannot be separated by continuous functions, inducing the functions of $C(X)$ on $X^{*}$ in the obvious manner, and then furnishing $X^{*}$ with the weakest topology in which these functions are continuous (see Theorem 3.9 in [27] for more details). This observation eliminates any reason for considering vector lattices of real-valued continuous functions on other than Tychonoff spaces. Therefore, it will be assumed henceforth that $X$ is a Tychonoff space unless the contrary is stated explicitly.

Hewitt's great paper [32] built on the aforementioned works of Stone and Cech and laid the foundation for the study of the interplay between $C(X)$ and $X$. In today's terminology the Tychonoff space $X$ is said to be realcompact if there is no strictly large Tychonoff space $Y$ such that $X$ is dense in $Y$ and every $f \in C(X)$ has an extension in $C(Y)$. Actually, Hewitt in [32] used the terminology $Q$-space instead of realcompact space and proved that $X$ is realcompact if and only if it is homeomorphic to a closed subspace of a product of real lines equipped with the usual product topology. This characterization is often used as a definition of realcompact spaces. Later, Shirota [52] showed that $X$ is realcompact if and only if to each algebra homomorphism $\varphi$ from $C(X)$ onto the real field $\mathbb{R}$ there corresponds a point $x$ of $X$ such that $\varphi(f)=f(x)$ for all $f \in C(X)$. This remarkable necessary and sufficient condition for 
a Tychonoff space to be realcompact was obtained very recently by Ercan and Önal [26] via an elementary approach. These observations will be used next to obtain an alternative characterization of realcompact spaces which is a little more fit for our study.

Lemma 3.1. Let $X$ be a Tychonoff space $X$. Then the following are equivalent.

(i) $X$ is realcompact

(ii) To each lattice homomorphism $\varphi$ from $C(X)$ onto $\mathbb{R}$ with $\varphi\left(\mathbf{1}_{X}\right)=1$ there corresponds a point $x$ of $X$ such that $\varphi(f)=f(x)$ for all $f \in C(X)$.

Proof. By the above Shirota's result, it suffices to prove that if $\varphi$ is a mapping of $C(X)$ to $\mathbb{R}$ with $\varphi\left(\mathbf{1}_{X}\right)=1$, then $\varphi$ is a lattice homomorphism if and only if $\varphi$ is an algebra homomorphism. So, let $\varphi$ be such a mapping and assume $\varphi$ to be an algebra homomorphism. If $f \in C(X)$ then

$$
0 \leq\left(\varphi\left(|f|^{1 / 2}\right)\right)^{2}=\varphi(|f|)=\left(\varphi\left(f^{2}\right)\right)^{1 / 2}=\left((\varphi f)^{2}\right)^{1 / 2}=|\varphi(f)| \text {. }
$$

It follows that $\varphi$ is a lattice homomorphism. Conversely, suppose that $\varphi$ is a lattice homomorphism and let $f, h \in C(X)$ such that $\varphi(h)=0$. For every $\varepsilon \in(0, \infty)$, the inequalities $\mathbf{0}_{X} \leq|f h| \leq$ $\varepsilon\left|f^{2} h\right|+\varepsilon^{-1}|h|$ hold in $C(X)$. Thus,

$$
0 \leq|\varphi(f h)| \leq \varepsilon \varphi\left(\left|f^{2} h\right|\right)+\varepsilon^{-1} \varphi(|h|)=\varepsilon \varphi\left(\left|f^{2} h\right|\right) .
$$

As $\varepsilon$ is arbitrary in $(0, \infty)$, we get $\varphi(f h)=0$. We derive that if $g \in C(X)$ then $\varphi\left(g-\varphi(g) \mathbf{1}_{X}\right)=0$. Therefore,

$$
\varphi(f g)=\varphi(f g)-\varphi\left(\left(g-\varphi(g) \mathbf{1}_{X}\right) f\right)=\varphi(f) \varphi(g) .
$$

So, $\varphi$ is an algebra homomorphism and we are done.

From now on, $Y$ stands for an arbitrary topological space. The cozero-set of a function $w \in C(Y)$ is the set

$$
\operatorname{coz}(w)=\{y \in Y: w(y) \neq 0\}
$$

Now, let $w \in C(Y)$ and $\tau$ be a function of $Y$ to $X$ which is continuous on $\operatorname{coz}(w)$. It is readily verified that the mapping $T$ from $C(X)$ into $C(Y)$ defined by

$$
T(f)(y)=w(y) f(\tau(y)) \quad \text { for all } f \in C(X) \text { and } y \in Y
$$

is an order bounded disjointness preserving operator. Such a mapping is usually called a weighted composition operator. Next, we 
discuss the question whether this implication is reversible. Surprisingly, this question has an affirmative answer if $X$ in addition is realcompact.

Theorem 3.2. Let $T$ be a mapping of $C(X)$ to $C(Y)$ and assume $X$ to be realcompact. Then the following are equivalent.

(i) $T$ is an order bounded disjointness preserving operator.

(ii) There exists $w \in C(Y)$ and a function $\tau$ of $Y$ into $X$ such that $\tau$ is continuous on $\mathrm{coz}(w)$ and

$T(f)(y)=w(y) f(\tau(y)) \quad$ for all $f \in C(X)$ and $y \in Y$.

Proof. Only necessity will be proved. Let $T$ be an order bounded disjointness preserving operator from $C(X)$ into $C(Y)$. Hence $T^{+}$is a lattice homomorphism from $C(X)$ into $C(Y)$ (see Theorem 2.2). Put $w_{+}=T^{+}\left(\mathbf{1}_{X}\right)$ and define for each $f \in C(X)$ the function $S(f) \in C\left(\operatorname{coz}\left(w_{+}\right)\right)$by

$$
S(f)(y)=\frac{T^{+}(f)(y)}{w_{+}(y)} \quad \text { for all } y \in \operatorname{coz}\left(w_{+}\right) .
$$

The mapping $S$ thus defined from $C(X)$ into $C\left(\operatorname{coz}\left(w_{+}\right)\right)$is linear, obviously. Moreover, if $y \in \operatorname{coz}\left(w_{+}\right)$then

$$
\begin{aligned}
\left(\delta_{y} \circ S\right)|f| & =S(|f|)(y)=\frac{T^{+}(|f|)(y)}{w_{+}(y)} \\
& =\frac{\left|T^{+}(f)(y)\right|}{w_{+}(y)}=|S(f)(y)|=\left|\left(\delta_{y} \circ S\right) f\right| .
\end{aligned}
$$

Hence, $\delta_{y} \circ S$ is a lattice homomorphism from $C(X)$ onto $\mathbb{R}$ with $\left(\delta_{y} \circ S\right)\left(\mathbf{1}_{X}\right)=1$. Lemma 3.1 yields that a point $x_{y}$ of $X$ can be found so that

$$
S(f)(y)=\left(\delta_{y} \circ S\right)(f)=f\left(x_{y}\right) \quad \text { for all } f \in C(X) .
$$

Let $\tau_{+}$be the mapping of $\operatorname{coz}\left(w_{+}\right)$to $X$ defined by $\tau_{+}(y)=x_{y}$ for all $y \in \operatorname{coz}\left(w_{+}\right)$. By Theorem 3.8 in [27], $\tau_{+}$is continuous. We get also

$$
T^{+}(f)(y)=w_{+}(y)\left(f \circ \tau_{+}\right)(y) \quad \text { for all } f \in C(X), y \in \operatorname{coz}\left(w_{+}\right) \text {. }
$$

On the other hand, let $f \in C(X)$ and $y \in Y$ such that $T^{+}\left(\mathbf{1}_{X}\right)(y)=$ 0 . Also, let $\varepsilon \in(0, \infty)$ and observe that

$$
\mathbf{0}_{X} \leq|f| \leq|f| \wedge \varepsilon \mathbf{1}_{X}+\varepsilon^{-1} f^{2} .
$$


Therefore,

$$
\begin{aligned}
0 & \leq\left|T^{+}(f)(y)\right| \\
& \leq\left|T^{+}(f)(y)\right| \wedge \varepsilon T^{+}\left(\mathbf{1}_{X}\right)(y)+\varepsilon^{-1} T^{+}\left(f^{2}\right)(y) \\
& =\varepsilon^{-1} T^{+}\left(f^{2}\right)(y) .
\end{aligned}
$$

So, $\left(T^{+}(f)\right)(y)=0$ because $\varepsilon$ is arbitrary in $(0, \infty)$. Now, choose an arbitrary extension of $\tau_{+}$to $Y$ and denote such an extension again by $\tau_{+}$. We derive directly that

$$
\left(T^{+}(f)\right)(y)=w_{+}(y) f\left(\tau_{+}(y)\right) \text { for all } f \in C(X) \text { and } y \in Y .
$$

Analogously, if $w_{-}=T^{-}\left(\mathbf{1}_{X}\right)$ then we may find a function $\tau_{-}$of $Y$ into $X$ such that $\tau_{-}$is continuous on $\operatorname{coz}\left(w_{-}\right)$and

$$
T^{-}(f)(y)=w_{-}(y) f\left(\tau_{-}(y)\right) \quad \text { for all } f \in C(X) \text { and } y \in Y .
$$

Observe now that

$$
w_{+} \wedge w_{-}=T^{+}\left(\mathbf{1}_{X}\right) \wedge T^{-}\left(\mathbf{1}_{X}\right)=\left(T\left(\mathbf{1}_{X}\right)\right)^{+} \wedge\left(T\left(\mathbf{1}_{X}\right)\right)^{-}=\mathbf{0}_{X}
$$

(where we use once more Theorem 2.2). But then

$$
\operatorname{coz}\left(w_{+}\right) \cap \operatorname{coz}\left(w_{-}\right)=\emptyset \quad \text { and } \quad \operatorname{coz}\left(w_{+}\right) \cup \operatorname{coz}\left(w_{-}\right)=\operatorname{coz}(w),
$$

where $w=w_{+}-w_{-} \in C(Y)$. Choose a function $\tau$ of $Y$ to $X$ so that $\tau(y)=\tau_{+}(y)$ if $y \in \operatorname{coz}\left(w_{+}\right)$and $\tau(y)=\tau_{-}(y)$ if $y \in \operatorname{coz}\left(w_{-}\right)$.

Since $T=T^{+}-T^{-}$, we derive that

$$
T(f)(y)=w(y) f(\tau(y)) \quad \text { for all } f \in C(X) \text { and } y \in Y
$$

and we are done.

Next, we shall say a few words to see that the condition of realcompactness imposed on the Tychonoff space $X$ in Theorem 3.2 is close to being the best possible for order bounded disjointness preserving operators from $C(X)$ into $C(Y)$ to be automatically weighted composition operators. Indeed, assume that $\mathbb{R}^{C(X)}$ is endowed with the usual product topology. The mapping $\pi$ of $X$ to $\mathbb{R}^{C(X)}$ defined by $\pi(x)(f)=f(x)$ for all $x \in X$ and $f \in C(X)$ sends $X$ homeomorphically to $\pi(X)=\{\pi(x): x \in X\}$ (see [27]). Let $v X$ denote the closure of $\pi(X)$ in $\mathbb{R}^{C(X)}$. Hence, $v X$ is the unique (up to a homeomorphism leaving $X$ pointwise fixed) realcompact topological space such that $X$ is dense in $v X$ and every function $f \in C(X)$ has a unique extension $f^{v} \in C(v X)$. The realcompact space $v X$ is 
referred to as the realcompactification of $X$ (see again [27]). Moreover, the mapping $v$ of $C(X)$ to $C(v X)$ defined by $v(f)=f^{v}$ for all $f \in C(X)$ is a lattice isomorphism as it was observed by Shirota in [52] and Henriksen [31].

At this point, assume that $X$ and $Y$ are both locally compact (and then Tychonoff). Recall that the set $C_{0}(X)\left(C_{\infty}(X)\right.$ in the book [27]) of all real-valued continuous functions on $X$ vanishing at infinity is a vector sublattice of $\mathbb{R}^{X}$. Meyer-Nieberg proved in [45] that a mapping $T$ of $C_{0}(X)$ to $C_{0}(Y)$ is a lattice homomorphism if and only if there exist a positive (real-valued) function $w$ on $Y$ which is continuous on $\operatorname{coz}(w)$ and a continuous function $\tau$ of $\operatorname{coz}(w)$ to $X$ such that, if $f \in C_{0}(X)$, then $T(f)(y)=w(y) f(\tau(y))$ if $y \in \operatorname{coz}(w)$ and $T(f)(y)=0$ of $y \notin \operatorname{coz}(w)$. In view of Theorem 2.2 , a same argument as previously used in the end of the proof of Theorem 3.2 leads straightforwardly to the following result.

Theorem 3.3. Assume that $X$ and $Y$ are locally compact and let $T$ be a mapping of $C_{0}(X)$ to $C_{0}(Y)$. Then the following are equivalent.

(i) $T$ is an order bounded disjointness preserving operator.

(ii) There exist a real-valued function $w$ on $Y$ which is continuous on $\operatorname{coz}(w)$ and a continuous function $\tau$ of $\operatorname{coz}(w)$ to $X$ such that, if $f \in C_{0}(X)$, then

$$
T(f)(y)=\left\{\begin{array}{l}
w(y) f(\tau(y)) \quad \text { if } y \in \operatorname{coz}(w) \\
0 \quad \text { if } y \notin \operatorname{coz}(w) .
\end{array}\right.
$$

At last, it should be pointed out that Theorem 3.3 was obtained in an alternative way by Jeang and Wong in [36]. Also, notice that Theorem 3.2 and Theorem 3.3 have the same compact version. Such a version has been obtained earlier by Arendt in [8] (see [35] by Jarosz for a different approach). In this regard, one might hope that Theorem 3.3 can be obtained from its compact version by extending the order bounded disjointness preserving operator $T$ from $C_{0}(X)$ into $C_{0}(Y)$ to an order bounded disjointness preserving operator $T^{\alpha}$ from $C(\alpha X)$ into $C(\alpha Y)$, where $\alpha X$ denotes the one-point compactification of $X$ (see [27]). However, Jeang and Wong provided in [36] the following example of an order bounded disjointness preserving operator $T$ from $C_{0}(X)$ into $C_{0}(Y)$ which does not have any such extension. 
Example 3.4. Let $X=[0, \infty)$ and $Y=\mathbb{R}$ with the usual topology and define $w, \tau$ from $\mathbb{R}$ into $\mathbb{R}$ by

$$
w(y)=\left\{\begin{array}{l}
1 \text { if } y>2 \\
y-1 \text { if } 0 \leq y \leq 2 \\
-1 \text { if } y<0
\end{array} \text { and } \tau(y)=\left\{\begin{array}{l}
y \text { if } y \geq 0 \\
-y \text { if } y<0 .
\end{array}\right.\right.
$$

Let $T$ be the mapping from $C_{0}(X)$ to $C_{0}(Y)$ defined by $T(f)(y)=$ $w(y \mathrm{f} \tau(y))$ for all $f \in C(X)$ and $y \in Y$. Clearly, $T$ is an order bounded disjointness preserving operator from $C_{0}(X)$ into $C_{0}(Y)$. But no extension $T^{a}$ from $C(\alpha X)$ into $C(\alpha Y)$ of $T$ can be an order bounded disjointness preserving operator.

\section{A Multiplicative Aspect}

In this section, we show how can results in Section 3 be extended to the more general setting of function algebras in the sense of Birkhoff and Pierce [15]. A vector lattice $L$ which is simultaneously an associative algebra such that $f g \in L^{+}$for all $f, g \in L^{+}$is called a lattice ordered algebras (briefly, an $\ell$-algebra). In [15], Birkhoff and Pierce called the $\ell$-algebra $L$ a function algebra (shortly, an $f$-algebra) if $(f h) \wedge g=(h f) \wedge g=0$ for all $f, g, h \in L^{+}$with $f \wedge g=0$. One of the most classical examples of $f$-algebras is $\mathbb{R}^{X}$ for any nonvoid set $X$. Moreover, if $X$ is a topological space, then $C(X)$ is a uniformly complete $f$-subalgebra of $\mathbb{R}^{X}$. In this space, we focus only on Archimedean $f$-algebras. The Archimedean $f$-algebra $L$ with a multiplicative unity is semiprime, meaning that, 0 is the only nilpotent element of $L$. Orthomorphisms on an Archimedean vector lattice $L$ is an important example of $f$-algebras. Indeed, the Archimedean vector lattice Orth $(L)$ is $f$-algebra with respect to the composition and the identity operator $I_{L}$ on $L$ is a multiplicative unity in $\operatorname{Orth}(L)$. On the other hand, if $L$ is an Archimedean semiprime $f$-algebra, then $L$ can be embedded in $\operatorname{Orth}(L)$ as an $f$-subalgebra. Below, we shall identify $L$ with an $f$-subalgebra of $\operatorname{Orth}(L)$ without further ado. The reader is referred to the surveys $[22,23]$ for more information on $f$-algebras. Also, Chapter 20 in [60] by Zaanen presents an excellent study of $f$-algebras based upon the Ph.D. thesis [48] of de Pagter. Next, we shall describe another important instance of $f$-algebras, namely, the $f$-algebra of all extended orthomorphisms on an Archimedean vector lattice. 
Let $L$ be an Archimedean vector lattice. Luxemburg and Schep in [40] defined an order bounded operator $T$ from an order dense order ideal $D_{T}$ of $L$ into $L$ to be an extended orthomorphism of $L$ if $|f| \wedge|g|=0$ in $D_{T}$ implies $|T f| \wedge|g|=0$ in $L$. Of course, an extended orthomorphism $T$ of $L$ is an orthomorphism of $L$ if $D_{T}=L$. A natural equivalence relation can be introduced in the set of all extended orthomorphisms of $L$ as follows. Two extended orthomorphisms of $L$ are equivalent whenever they agree on an order dense order ideal in $L$ or, equivalently, they are equal on the intersection of their domains. Notice that the intersection of two order dense order ideals in $L$ is obviously again an order dense order ideal in $L$. The set of all equivalence classes of extended orthomorphisms of $L$ is denoted by $\operatorname{Orth}^{\infty}(L)$. With respect to the pointwise addition, scalar multiplication, and ordering, $\operatorname{Orth}^{\infty}(L)$ is an Archimedean vector lattice. The lattice operations in the vector lattice $\operatorname{Orth}^{\infty}(L)$ are given pointwise. It turns out that the vector lattice $\operatorname{Orth}^{\infty}(L)$ is an $f$-algebra under the composition as multiplication. Moreover, since extended orthomorphisms are order continuous, the set Orth $(L)$ of all orthomorphisms of $L$ can be embedded naturally in $\operatorname{Orth}^{\infty}(L)$ as an $f$-subalgebra. Obviously, the identity operator $I_{L}$ of $L$ serves as a multiplicative unity in $\operatorname{Orth}^{\infty}(L)$. All these facts can be found in the fundamental papers [40] by Luxemburg and Schep and [49] by de Pagter.

As previously pointed out, this section gives a look at concrete situations presented in Section 3 from a 'purely algebraic' point of view. In this prospect, some extra observations are needed. Let $X$ be a realcompact space and $Y$ be a Tychonoff space. It is shown in Theorem 3.2 that if $T$ is an order bounded disjointness preserving operator from $C(X)$ into $C(Y)$ then there exist $w \in C(Y)$ and a function $\tau$ of $Y$ into $X$ which is continuous on $\operatorname{coz}(w)$ such that $T(f)(y)=w(y) f(\tau(y))$ for all $f \in C(X)$ and $y \in Y$. The observation to make here is that if $f$ is in $C(X)$ then the real-valued function $S(f)$ defined on $Y$ by $S(f)(y)=f(\tau(y))$ for all $y \in Y$ need not be in $C(Y)$. However, it is readily verified that $S(f)$ is continuous on some dense open set in $Y$. In other words, $S(f)$ belongs to $\operatorname{Orth}^{\infty}(C(Y))$. Indeed, $\operatorname{Orth}^{\infty}(C(Y))$ is essentially the algebra of all continuous functions defined on some dense open set of $Y$ (see [29] by Hager, [49] by de Pagter, and [57] by Wickstead). Accordingly, the mapping $S$ defined from $C(X)$ into $\mathbb{R}^{Y}$ by $S(f)=f \circ \tau$ for all $f \in C(X)$ is actually a lattice and algebra homomorphism from 
$C(X)$ into $\operatorname{Orth}^{\infty}(C(Y))$. Summarizing, Theorem 3.2 can be stated algebraically as follows. A mapping $T$ from $C(X)$ into $C(Y)$ is an order bounded disjointness preserving operator if and only if there exist $w \in C(Y)$ and a lattice and algebra homomorphism $S$ from $C(X)$ into $\operatorname{Orth}^{\infty}(C(Y))$ such that $T(f)(y)=w(y)(S(f))(y)$ for all $f \in C(X)$ and $y \in Y$. It seems to be natural therefore to ask whether this 'algebraic' version of Theorem 3.2 can be extended to the more general setting of $f$-algebras. From now on, $A$ and $B$ stand for Archimedean semiprime $f$-algebras with $B$ uniformly complete.

Theorem 4.1. Assume A to have a multiplicative unity and let $T$ be a mapping from $A$ into $B$. Then the following are equivalent.

(i) $T$ is an order bounded disjointness preserving operator.

(ii) There exist $w \in B$ and a lattice and algebra homomorphism from $A$ into $\operatorname{Orth}^{\infty}(B)$ such that $T(f)=w S(f)$ for all $f \in A$.

Next, we focus on Theorem 3.3 in which we have seen that if $X$ and $Y$ are locally compact spaces and $T$ is an order bounded disjointness preserving operator from $C_{0}(X)$ into $C_{0}(Y)$, then there exist a real-valued function $w$ on $Y$ which is continuous on $\operatorname{coz}(w)$ and a continuous function $\tau$ of $\operatorname{coz}(w)$ to $X$ such that, if $f \in C_{0}(X)$, then $T(f)(y)=w(y) f(\tau(y))$ if $y \in \operatorname{coz}(w)$, and $T(f)(y)=0$ if $y \notin \operatorname{coz}(w)$. In [17], it shown that $C_{b}(Y)$ and $\operatorname{Orth}\left(C_{0}(Y)\right)$ are isomorphic as $f$-algebras. Also, the $f$-algebras $\operatorname{Orth}^{\infty}\left(C_{b}(Y)\right)$ and $\operatorname{Orth}^{\infty}(C(Y))$ are isomorphic (see again $\left.[29,49,57]\right)$. Hence, the above result can be stated as follows. A mapping $T$ from $C_{0}(X)$ into $C_{0}(Y)$ is an order bounded disjointness preserving operator if and only if there exist $w \in \operatorname{Orth}^{\infty}\left(C_{b}(Y)\right)$ and a lattice and algebra homomorphism $S$ from $C(X)$ into $\operatorname{Orth}^{\infty}\left(C_{b}(Y)\right)$ such that $T(f)(y)=w(y)(S(f))(y)$ for all $f \in C_{0}(X)$ and $y \in Y$. This result holds in $f$-algebras as we shall see next. First of all, the $f$-algebra $A$ is said to be $n^{\text {th }}$-root closed for some nonzero natural number $n$ if for every $g \in A^{+}$there exists $f \in A^{+}$such that $f^{n}=g$ (such an $f$ is unique since $A$ is assumed to be semiprime). The proof of the following theorem can be found in the recent survey [23].

Theorem 4.2. Assume that $A$ is $n^{\text {th }}$-root closed for some positive integer and let $T$ be a mapping from $A$ into $B$. Then the following are equivalent.

(i) $T$ is an order bounded disjointness preserving operator. 
(ii) There exist $w \in \operatorname{Orth}^{\infty}(\operatorname{Orth}(B))$ and a lattice and algebra homomorphism $S$ from $A$ into $\operatorname{Orth}^{\infty}(\operatorname{Orth}(B))$ such that $T(f)=w S(f)$ for all $f \in A$.

The proof of Theorem 4.2 presented in [23] is based upon a beautiful theorem by Hart [30], namely, if $L$ and $M$ are Archimedean vector lattices and $T$ is an order bounded disjointness preserving operator $T$ from $L$ into $M$, then there exists a lattice and algebra homomorphism $\widetilde{T}$ from $\operatorname{Orth}(L)$ into $\operatorname{Orth}(\mathcal{R}(T(L))$ ) (where $\mathcal{R}(T(L))$ is the vector sublattice of $M$ generated by $T(L))$ such that $\widetilde{T}(S)(T(f))=T(S(f))$ for all $T \in \operatorname{Orth}(L)$ and $f \in L$.We end this section with an example (see [23]) showing that the condition imposed on $A$ in Theorem 4.2 cannot be deleted.

Example 4.3. Let $A$ be the $f$-algebra of the piecewise polynomial functions on $[0,1]$ that are 0 at 0 . Then the real-valued lattice homomorphism $T$ on $A$ that assigns to a function its right derivative at 0 is not representable as in the main theorem above. Indeed, denote the identity function on $[0,1]$ by $f$. Suppose that $T$ has a representation as above with $S$ a lattice and algebra homomorphism from $A$ onto $\mathbb{R}$ and $\alpha$ a nonzero real number such that $T=\alpha S$. Then $S(f) \neq 0$, hence $S\left(f^{2}\right) \neq 0$, but $T\left(f^{2}\right)=0$, a contradiction.

\section{A Global Point of View}

In this section, we look at disjointness preserving operators from a certain 'global' point of view. Indeed, we focus on the lattice structure of certain sets of operators preserving disjointness rather that the behavior of the disjointness preserving operators themselves. We start our investigation by introducing the notion of disjointness preserving sets. A nonvoid subset $\mathcal{D}$ of $\mathcal{L}_{b}(L, M)$ is called a disjointness preserving set in $\mathcal{L}_{b}(L, M)$ if $|S(f)| \wedge|T(g)|=0$ for all $S, T \in \mathcal{D}$ and $f, g \in L$ with $|f| \wedge|g|=0$. Several elementary properties follow straightforwardly from the definition. Let us single out a few as particularly worthy. For instance, an order bounded operator $T$ from $L$ into $M$ preserve disjointness if and only if $\{T\}$ is a disjointness preserving set in $\mathcal{L}_{b}(L, M)$. Therefore, any element in a disjointness preserving set in $\mathcal{L}_{b}(L, M)$ is an order bounded disjointness preserving operator. Moreover, the non-void subset $\mathcal{D}$ of $\mathcal{L}_{b}(L, M)$ is a disjointness preserving set in $\mathcal{L}_{b}(L, M)$ if and only if each pair $\{S, T\}$ of elements of $\mathcal{D}$ is a disjointness preserving set in $\mathcal{L}_{b}(L, M)$. 
Thus, if $\mathcal{D}$ is a disjointness preserving set in $\mathcal{L}_{b}(L, M)$ then so is any nonvoid subset of $\mathcal{D}$. However, the next property of disjointness preserving sets is not so visible at first sight. Indeed, it turns out that each pair in a disjointness preserving set in $\mathcal{L}_{b}(L, M)$ has a supremum and an infimum in $\mathcal{L}(L, M)$. More details are given in the following.

Lemma 5.1. Let $\mathcal{D}$ be a disjointness preserving set in $\mathcal{L}_{b}(L, M)$. Then each pair $\{S, T\}$ of elements of $\mathcal{D}$ has a supremum $S \vee T$ and a infimum $S \wedge T$ in $\mathcal{L}(L, M)$ such that

$$
(S \vee T)(f)=S(f) \vee T(f) \text { and }(S \wedge T)(f)=S(f) \wedge T(f)
$$

for all $f \in L^{+}$.

Proof. Let $S, T \in \mathcal{D}$ and $f, g \in L$ such that $|f| \wedge|g|=0$. Since $\mathcal{D}$ is a disjointness preserving set in $\mathcal{L}_{b}(L, M)$, the sets $\{S(f), T(f)\}$ and $\{S(g), T(g)\}$ are disjoint. Hence,

$$
0 \leq|S(f)-T(f)| \wedge|S(g)-T(g)|=0
$$

So, the difference $S-T$ is an order bounded disjointness preserving operator from $L$ into $M$. By Theorem 2.2, $S-T$ has an absolute value $|S-T|$ in the ordered vector space $\mathcal{L}(L, M)$ such that

$$
|S-T|(f)=|S(f)-T(f)| \quad \text { for all } f \in L^{+} .
$$

This yields quickly that the pair $\{S, T\}$ has a least upper bound $S \vee T$ and a great lower bound $S \wedge T$ in $\mathcal{L}(L, M)$ given by

$$
S \vee T=\frac{1}{2}(S+T+|S-T|) \quad \text { and } \quad S \wedge T=\frac{1}{2}(S+T-|S-T|)
$$

Now, we prove that these supremum and infimum are given pointwise. On the other hand, if $f \in L^{+}$then

$$
\begin{aligned}
(S \vee T)(f) & =\left(\frac{1}{2}(S+T+|S-T|)\right)(f) \\
& =\frac{1}{2}(S(f)+T(f)+|S-T|(f)) \\
& =\frac{1}{2}(S(f)+T(f)+|S(f)-T(f)|)=S(f) \vee T(f) .
\end{aligned}
$$

The formula

$$
(S \wedge T)(f)=S(f) \wedge T(f) \quad \text { for all } f \in L^{+}
$$

is obtained in the same way completing the proof of the lemma. 
Now, let $S, T$ be order bounded disjointness preserving operators from $L$ into $M$. A short's moment thought (see the first lines of the previous proof) reveals that if $\{S, T\}$ is a disjointness preserving set in $\mathcal{L}_{b}(L, M)$ then the sum $S+T$ preserves disjointness. The question wether this implication is reversible is discussed next.

Lemma 5.2. Let $S, T$ be order bounded disjointness preserving operators from $L$ into $M$. Then the following are equivalent.

(i) $S+T$ preserves disjointness

(ii) The pair $\{S, T\}$ is a disjointness preserving set in $\mathcal{L}_{b}(L, M)$.

Proof. Only necessity is proved. So, assume $S+T$ to be disjointness preserving. By Theorem 2.2, the absolute value $|S+T|$ exists in the ordered vector space $\mathcal{L}(L, M)$. Furthermore, if $f \in L^{+}$then

$0 \leq|(|S|-|T|)(f)|=|S(f)|-|T(f)| \leq|S(f)+T(f)|=|S+T|(f)$. It follows readily that $|S|-|T|$ preserves disjointness. So, the absolute value ||$S|-| T||$ of $|S|-|T|$ exists in $\mathcal{L}(L, M)$ and if $f \in L^{+}$ then

$$
|| S|-| T||(f)=|| S|(f)-| T|(f)|=|| S(f)|-| T(f)|| .
$$

As in the proof of Lemma 5.1, the pair $\{|S|,|T|\}$ has a supremum $|S| \vee|T|$ and a infimum $|S| \wedge|T|$ in $\mathcal{L}(L, M)$. Moreover, if $f \in L^{+}$ then

$(|S| \vee|T|)(f)=|S(f)| \vee|T(f)|$ and $(|S| \wedge|T|)(f)=|S(f)| \wedge|T(f)|$. At this point, let $f, g \in L$ such that $|f| \wedge|g|=0$. Since $|S|$ and $|T|$ are lattice homomorphisms from $L$ into $M$, we can write

$$
\begin{aligned}
(|S| \vee|T|)(|f|)+(|S| \vee|T|)(|g|) & =(|S| \vee|T|)(|f|+|g|) \\
& =(|S| \vee|T|)(|f| \vee|g|) \\
& =(|S|(|f| \vee|g|)) \vee(|T|(|f| \vee|g|)) \\
& =|S| f|| \vee|S| g|| \vee|T| f|| \vee|T| g|| \\
& =|S| f|| \vee|T| f|| \vee|S| g|| \vee|T| g|| \\
& =(|S| \vee|T|)(|f|) \vee(|S| \vee|T|)(|g|) .
\end{aligned}
$$

Thus,

$$
\begin{aligned}
0 & \leq|S(f)| \wedge|T(g)| \\
& =|| S|(f)| \wedge|| T|(g)|=|S(f)| \wedge|T(g)| \\
& \leq(|S| \vee|T|)(|f|) \wedge(|S| \vee|T|)(|g|)=0 .
\end{aligned}
$$


We derive that $\{S, T\}$ is a disjointness preserving set and we are done.

In [13], Bernau, Huijsmans, and de Pagter studied sums of order bounded disjointness preserving operators and gave various properties of such sums. More recently in [50], de Pagter in collaboration with Schep furnished several necessary and sufficient conditions for a sum of two order bounded disjointness preserving operators in order to be again preserving disjointness. Lemma 5.2 above can be seen as a modest contribution to this study.

Now, recall that the set $\operatorname{Orth}(M)$ of all orthomorphisms on $M$ is a generalized vector sublattice of $\mathcal{L}(M)$ the lattice operations of which are given pointwise. Actually, this nice property of $\operatorname{Orth}(M)$ has something to do with the fact that $\operatorname{Orth}(M)$ is a particular disjointness preserving set in $\mathcal{L}_{b}(M)$. Let us say some additional words in order to explain our point of view. Assume that $\mathcal{D}$ is a disjointness preserving set in $\mathcal{L}_{b}(M)$ that contains $\operatorname{Orth}(M)$. Hence, $I_{M} \in \mathcal{D}$ and $\left\{I_{M}, T\right\}$ is a disjointness preserving set in $\mathcal{L}_{b}(M)$ for all $T \in \mathcal{D}$. It follows that $T$ is an orthomorphism on $M$, that is, $\mathcal{D}=\operatorname{Orth}(M)$. In other words, $\operatorname{Orth}(M)$ is a maximal element in the set of all disjointness preserving sets in $\mathcal{L}_{b}(M)$ with respect to the inclusion ordering. Surprisingly, it turns out that any maximal disjointness preserving set in $\mathcal{L}_{b}(L, M)$ is a generalized vector sublattice of $\mathcal{L}(L, M)$. To see this, let us define a disjointness preserving set $\mathcal{M}$ in $\mathcal{L}_{b}(L, M)$ to be maximal if there is no strictly large disjointness preserving set in $\mathcal{L}_{b}(L, M)$. We are in position now to prove the central theorem of this section.

Theorem 5.3. Let $\mathcal{M}$ be a maximal disjointness preserving set in $\mathcal{L}_{b}(L, M)$. Then $\mathcal{M}$ is a generalized vector sublattice of $\mathcal{L}(L, M)$. Moreover, if $S, T \in \mathcal{M}$ then

$$
(S \vee T)(f)=S(f) \vee T(f) \text { and }(S \wedge T)(f)=S(f) \wedge T(f)
$$

for all $f \in L^{+}$.

Proof. Let $S, T \in \mathcal{M}$ and $a$ be a real number. Since $\{S, T\}$ is a disjointness preserving set in $\mathcal{L}_{b}(L, M)$, Lemma 5.2 yields that the sum $S+T$ preserves disjointness. Now, let $R \in \mathcal{M}$ and $f, g \in L$ such that $|f| \wedge|g|=0$. Hence, $\{R, S, T\}$ is a disjointness preserving set in $\mathcal{L}_{b}(L, M)$ so

$$
|R(f)| \wedge|S(g)|=|R(f)| \wedge|T(g)|=0
$$


We derive that

$$
0 \leq|R(f)| \wedge|(S+T)(g)| \leq|R(f)| \wedge(|S(g)|+|T(g)|)=0 .
$$

Thus, $\{R, S+T\}$ is a disjointness preserving set in $\mathcal{L}_{b}(L, M)$. It follows easily that $\mathcal{M} \cup\{S+T\}$ is again a disjointness preserving set in $\mathcal{L}_{b}(L, M)$. Since $\mathcal{M}$ is maximal, $S+T \in \mathcal{M}$. Analogously, it is readily checked that $\mathcal{M} \cup\{a T\}$ is a disjointness preserving set in $\mathcal{L}_{b}(L, M)$ and then, by maximality, $a T \in \mathcal{M}$. This implies that $\mathcal{M}$ is a vector subspace of $\mathcal{L}(L, M)$.

At this point, let $T \in \mathcal{M}$. Since $T$ is an order bounded disjointness preserving operator from $L$ into $M$, the absolute value $|T|$ of $T$ in $\mathcal{L}(L, M)$ exists (where we use Theorem 2.2). Let $S \in \mathcal{M}$ and $f, g \in L$ such that $|f| \wedge|g|=0$. Theorem 2.2 together with the fact that $\mathcal{M}$ is a disjointness preserving set in $\mathcal{L}_{b}(L, M)$ leads to

$$
|R(f)| \wedge|| T|(g)|=|R(f)| \wedge|T(g)|=0 .
$$

We derive that $\mathcal{M} \cup\{|T|\}$ is a disjointness preserving set in $\mathcal{L}_{b}(L, M)$. Since $\mathcal{M}$ is maximal as a disjointness preserving set in $\mathcal{L}_{b}(L, M)$, we get $\mathcal{M}=\mathcal{M} \cup\{|T|\}$ so $|T| \in \mathcal{M}$. It follows that $\mathcal{M}$ is a generalized vector sublattice of $\mathcal{L}(L, M)$ and we are done.

Alternative aspects of maximal disjointness preserving sets can be found in the recent works $[10,11]$. For instance, this concept is used in [11] to give an elementary proof of the existence of the modulus of complex order bounded disjointness preserving operators between two arbitrary complex vector lattices. This fact was first proved via Zorn's Lemma by Meyer in [42] for uniformly complete complex vector lattices. More recently, Grobler and Huijsmans obtained the Meyer result constructively [28]. In [11], the result was proved without assuming the complex vector lattices under consideration to be uniformly complete. Another property of maximal disjointness preserving set in $\mathcal{L}_{b}(L, M)$ is discussed next.

Wickstead proved in [56] if $L$ in addition is Dedekind complete, then $\operatorname{Orth}(L)$ is a band of the vector lattice $\mathcal{L}_{b}(L)$. This result is extended in what follows to more general setting of maximal disjointness preserving sets. We should recall here that if $M$ is Dedekind complete, then the ordered vector space $\mathcal{L}_{b}(L, M)$ is a Dedekind complete vector lattice. The following proposition follows from Proposition 2.2 in [10] . 
Proposition 5.4. If $M$ is Dedekind complete, then any maximal disjointness preserving set in $\mathcal{L}_{b}(L, M)$ is a band of $\mathcal{L}_{b}(L, M)$.

Now, assume that $L$ has a strong order unit $e>0$ (that is, the inequality $|f| \leq a|e|$ holds for all $f \in L$ and some real number $a$ ) and let $\mathcal{M}$ be a order ideal of $\mathcal{L}_{b}(L, M)$. Define the positive operator $\Pi_{e}$ from $\mathcal{M}$ into $M$ by

$$
\Pi_{e}(T)=T(e) \quad \text { for all } T \in \mathcal{M} .
$$

The following result is a direct inference of Theorem 3.3 in [10].

Theorem 5.5. Assume $L$ to have a strong order unit e and $M$ to be Dedekind complete. If $\mathcal{M}$ is an order ideal of $\mathcal{L}_{b}(L, M)$ then the following are equivalent.

(i) $\mathcal{M}$ is a maximal disjointness preserving set in $\mathcal{L}_{b}(L, M)$.

(ii) $\Pi_{e}$ is a lattice isomorphism.

(iii) $\Pi_{e}$ is bijective.

In particular, if $L$ has a strong order unit $e$ and $M$ is Dedekind complete, then $\mathcal{L}_{b}(L, M)$ has a unique (up to a lattice isomorphism) maximal disjointness preserving set, which is a vector lattice copy of $M$. This fact turns out to be an extension of a classical fact due to Zaanen in [58], namely, if $M$ is a Dedekind complete vector lattice with a strong order unit, then $\operatorname{Orth}(M)$ and $M$ are isomorphic as vector lattices. Actually, Zaanen proved a stronger result, viz., Orth $(M)$ and $M$ are isomorphic as vector lattices as soon as $M$ is uniformly complete and has a strong order unit. It seems to be natural therefore to ask the following question.

Problem 5.6. Do the equivalences in Theorem 5.5 hold if $M$ is only uniformly complete?

The next paragraph deals with maximal disjointness preserving sets on certain $C(X)$-spaces. First of all, let $X$ and $Y$ be topological spaces and let $\tau$ be a function of $Y$ to $X$. For every $w \in C(Y)$, let $C_{\omega, \tau}$ indicate the mapping from $C(X)$ into $\mathbb{R}^{Y}$ defined by

$$
C_{w, \tau}(f)(y)=w(f) f(\tau(y)) \quad \text { for all } f \in C(X) \text { and } y \in Y .
$$

Moreover, put

$$
\Omega_{\tau}=\left\{w \in C(Y): C_{\omega, \tau}(f) \in C(Y) \quad \text { for all } f \in C(X)\right\}
$$

and

$$
O_{\tau}=\cup_{w \in \Omega_{\tau}} \operatorname{coz}(w) .
$$


Obviously, $O_{\tau}$ is an open set in $Y$. In [9], Benamor observes that $\tau$ is continuous on $O_{\tau}$ and defines $\tau$ to be maximal if there is no large open set in $Y$ on which $\tau$ is continuous. A sleight modification of the proof of Corollary 1 in [9] yields directly to the following characterization of maximal disjointness preserving sets in $\mathcal{L}_{b}(C(X), C(Y))$ when $X$ in addition is compact.

Theorem 5.7. Assume that $X$ is a compact space and let $\mathcal{M}$ be a non-void set of $\mathcal{L}_{b}(C(X), C(Y))$. Then the following are equivalent.

(i) $\mathcal{M}$ is a maximal disjointness preserving set.

(ii) There exists a maximal function $\tau$ from $Y$ into $X$ such that $\mathcal{M}=\left\{C_{w, \tau}: w \in \Omega_{\tau}\right\}$.

At last, a careful examination of Theorem 5.7 leads naturally to the following open problem.

Problem 5.8. Let $T$ be a lattice homomorphism from $L$ into $M$ and put

$$
\mathcal{D}(T)=\{S \circ T: T \in \operatorname{Orth}(M)\} .
$$

It is not hard to see that $\mathcal{D}$ is a disjointness preserving set in $\mathcal{L}_{b}(L, M)$. Under what conditions is $\mathcal{D}$ maximal? Conversely, if such conditions are satisfied and $\mathcal{D}$ is an arbitrary maximal disjointness preserving set in $\mathcal{L}_{b}(L, M)$. Does there exist a lattice homomorphism $T$ from $L$ into $M$ such that $\mathcal{D}=\mathcal{D}(T)$ ?

\section{Algebraic Disjointness Preserving Operators}

Consider a square matrix $T$ for which on every row there is at most one nonzero entry. Let $n$ be the degree of its minimal polynomial and let $m$ be its valuation, that is, the multiplicity of 0 as a root of that minimal polynomial. Then $T^{n !}$ is diagonal, when restricted to the range of $T^{m}$. The latter looks surprising and one suspects that the result is known, but we have not been able to locate a reference for it. In this section we offer a wide ranging generalization of this matrix result. The condition above simply states that the matrix represents an operator that preserves disjointness in the pointwise ordering. The question arises naturally, as to whether general operators on vector lattices that preserve disjointness behave in a similar 
fashion. For obvious reasons, when leaving the domain of finite dimensional vector spaces, some form or another of continuity is reasonably imposed on the operators considered. Thus we consider order bounded disjointness preserving operators on Archimedean vector lattices. Fortunately, there is a concept of diagonal in vector lattices such that, surprisingly, order bounded disjointness preserving operators that satisfy a polynomial equation do behave like the matrix case. Indeed, the algebraic orthomorphisms serve the role of diagonals. This brings us to the main topic of the present section, algebraic order bounded disjointness preserving operators. Let us recall some of the relevant notions.

First, we recall the reader that $L$ is an Archimedean vector lattice. As usual, $\mathbb{R}[X]$ indicates the ring of all polynomials with coefficients in the real field $\mathbb{R}$. An operator $T$ on $L$ is said to be algebraic if $\Pi(T)=0$ for some nonzero polynomial $\Pi \in \mathbb{R}[X]$. Hence, $T \in \mathcal{L}(L)$ is algebraic if and only if the ring ideal

$$
\mathcal{I}(T)=\{\Pi \in \mathbb{R}[X]: \Pi(T)=0\}
$$

is not equal to $\{0\}$. Let $T \in \mathcal{L}(T)$ be an algebraic operator. Since the ring $\mathbb{R}[X]$ is principal, the ring ideal $\mathcal{I}(T)$ is generated by a unique monic polynomial $\Pi_{T}$, usually called the minimal polynomial of $T$. In particular, if $\Pi \in \mathbb{R}[X]$ then $\Pi(T)=0$ if and only if $\Pi_{T}$ divides $\Pi$. The notion of algebraic operators has been introduced by Kaplansky [37] for operators on Banach spaces.

Now, we say that an operator $T$ on $L$ is strongly diagonal if there exist pairwise disjoint components $P_{1}, P_{2}, \ldots, P_{m}$ of the identity operator $I_{I}$ and real numbers $\alpha_{1}, \alpha_{2}, \ldots, \alpha_{n}$ such that

$$
T=\alpha_{1} P_{1}+\alpha_{2} P_{2}+\cdots+\alpha_{m} P_{m} .
$$

Recall here that by a component of $I_{L}$ we mean a positive orthomorphism $P$ such that the equality $P \wedge\left(I_{L}-P\right)=0$ holds in the vector lattice Orth $(L)$ of all orthomorphisms on $L$. Strongly diagonal operators are usually called $I_{L^{-}}$step functions $[6,59]$. Clearly, a strongly diagonal operator on $L$ is an orthomorphism. It turns out that the converse holds if the orthomorphism under consideration is algebraic (see Theorem 3.3 in [21]).

Proposition 6.1. Let $T$ an operator on $L$. Then the following are equivalent.

(i) $T$ is a strongly diagonal

(ii) $T$ is an algebraic orthomorphism. 
The proof of the main result of this section is based upon the classical Kakutani-Bohnenblust-Krein representation theorem [1] and the representation of order bounded disjointness preserving operators on $C(X)$-spaces as weighted composition operators (see Section 3 ). However, the proof also uses the following lemma dealing with the existence of invariant principal order ideals, which is of independent interest (see Lemma 5.2 in [21]). First, recall that if $T \in \mathcal{L}(L)$ then a subset $D$ of $L$ is $T$-invariant if $T$ sends $D$ to $D$. Also, recall that an order ideal of $L$ which is generated by one element is said to be principal.

Lemma 6.2. Let $T$ be an algebraic order bounded disjointness preserving operator on $L$. For every $f \in L$ there exists a $T$-invariant principal order ideal of $L$ containing $f$.

We are in position now to state the central result of this section. The details can be found in Theorem 5.3 in [21].

Theorem 6.3. Let $T$ be an order bounded disjointness preserving operator on $L$. Then the following are equivalent.

(i) $T$ is algebraic.

(ii) There exist natural numbers $m$ and $n$ with $n>m$ such that the restriction of $T^{n !}$ to the vector sublattice of $L$ generated by the range $T^{m}(L)$ of $T^{m}$ is strongly diagonal.

Furthermore, when $T$ is algebraic, $n$ (respectively, $m$ ) can be chosen as the the degree (respectively, the valuation) of the minimal polynomial of $T$.

Once we observe that $\left|T^{n}\right|=|T|^{n}$ for all natural number $n$ and each order bounded disjointness preserving operator $T$ on $L$, it follows quickly from Theorem 6.3 that the absolute value of an algebraic order bounded disjointness preserving operator is algebraic as well. This seems far from obvious without the representation in Theorem 6.3 and contrasts with the fact that the absolute value of a finite rank operator need not be a finite rank operator (see [1]). On the other hand, in the above theorem we really need both $n$ and $m$, that is to say, it is possible that $T^{n}$ is not an orthomorphism on $L$ for any $n$. The following simple example illustrates that fact, whereas special cases where one can take $m=0$ will be discussed next.

Example 6.4. Let $L$ be the Archimedean vector lattice $\mathbb{R}^{2}$ with coordinatewise addition, scalar multiplication, and ordering and define 
$T \in \mathcal{L}(L)$ by $T(x, y)=(x, x)$ for all $(x, y) \in \mathbb{R}^{2}$. Clearly, $T$ is an order bounded disjointness preserving which is not an orthomorphism. Now, observe that $T^{n}=T$ for all $n \in \mathbb{N}$.

In [18], it is shown that if $T$ is an algebraic operator on a vector space, then $T$ is injective if and only if $T$ is surjective. Combining this fact with Theorem 6.3 we get easily the following.

Corollary 6.5. Let $T$ be a surjective (or injective) order bounded disjointness preserving operator from $L$ into $M$. Then the following are equivalent.

(i) $T$ is algebraic.

(ii) There exists a natural number $n$ such that $T^{n !}$ is a strongly diagonal.

Furthermore, $n$ can be chosen to be the degree the minimal polynomial of $T$.

At this point, we turn our attention to locally algebraic disjointness preserving operators. First of all, recall that the operator $T$ on the vector space $L$ is said to be locally algebraic if for every $f \in L$ there exists a nonzero polynomial $\Pi \in \mathbb{R}[X]$ (depending on $f$ ) such that $\Pi(T) f=0$ (see again [37] by Kaplansky). Obviously, any algebraic operator is locally algebraic. Next, we present a characterization of locally algebraic orthomorphism (see [21] for the proof).

Proposition 6.6. Let $T$ an orthomorphism on $L$. Then the following are equivalent.

(i) $T$ is locally algebraic.

(ii) The restriction of $T$ to each principal band of $L$ is algebraic (or strongly diagonal).

On the other hand, recently in [21], the notion of Kaplansky complete vector lattice was introduced as follows. The vector lattice $L$ is said to be Kaplansky complete if for every infinite countable subset $D$ of $L$ there exists $f \in L$ and an infinite subset $G$ of $D$ such that $f \wedge g=0$ for all $g \in G$. For instance, Banach lattices and vector lattices with weak order units are Kaplansky complete. This concept turns out to be crucial for understanding when every locally algebraic orthomorphism is strongly diagonal. For details, we refer to $[21]$. 
Theorem 6.7. The following are equivalent.

(i) $L$ is Kaplansky complete.

(ii) Every locally algebraic orthomorphism on $L$ is algebraic (and then a strongly diagonal operator).

As a consequence, we conclude that, for $\sigma$-Dedekind complete vector lattices, the condition that every orthomorphism is strongly diagonal is very strong as we can see next (see [21] for the proof).

Corollary 6.8. If $L$ is $\sigma$-Dedekind complete, then the following are equivalent.

(i) Every orthomorphism on $L$ is a strongly diagonal operator.

(ii) $L$ is finite dimensional.

The condition of Dedekind $\sigma$-completeness is not superfluous as it can be seen via the next Zaanen's example [58].

Example 6.9. Let $L$ be the Archimedean vector lattice of all realvalued continuous functions on $[0,1]$ which are piecewise linear. So, $L$ is not $\sigma$-Dedekind complete and $\operatorname{Orth}(L)=\left\{a I_{L}: a \in \mathbb{R}\right\}$.

Finally, it seems to be natural now to ask for the corresponding versions of Proposition 6.6 and Theorem 6.7 for order bounded disjointness preserving operators on the vector lattice $L$.

Problem 6.10. As for locally algebraic orthomorphisms, can a characterization of locally algebraic order bounded disjointness preserving operators be obtained in terms of algebraic operators?

Problem 6.11. What could be a necessary and sufficient condition on $L$ for locally algebraic order bounded disjointness preserving operators on $L$ to be algebraic?

\section{REFERENCES}

[1] Y. A. Abramovich, C. D. Aliprantis, An Invitation to Operator Theory, Grad. Stud. Math., vol. 50, Amer. Math. Soc., Providence, RI, 2002.

[2] Y. A. Abramovich, E. L. Arensen and A. K. Kitover, Banach $C(K)$-modules and Operators Preserving Disjointness, Pitman Research Notes in Mathematics Series, 277, Longman Scientific \& Technical, Harlow, 1992.

[3] Y. A. Abramovich, and A. K. Kitover, Inverses of disjointness preserving operators, Memoirs Amer. Math. Soc., 143 (2000), no 679.

[4] Y. A. Abramovich, A. I. Veksler, and A. V. Koldunov, Operators preserving disjointness, Dokl. Akad. Nauk., 248 (1979), 1033-1036.

[5] Y. A. Abramovich and A. W. Wickstead, Recent results on the order structure of compact operators, Irish Math. Soc. Bull., 32 (1994), 32-45. 
[6] C. D. Aliprantis, O. Burkinshaw, Positive Operators, Academic Press, Orlando, 1985.

[7] J. Araujo, E. Beckenstein and L. Narici, Biseparating maps and homeomorphic real-compactifications, J. Math. Ana. Appl., 12 (1995), 258-265.

[8] W. Arendt, Spectral properties of Lamperti operators, Indiana Univ. Math. J., 32 (1983), 199-215.

[9] F. Benamor, Riesz spaces of order bounded disjointness preserving operators, Comment. Math. Univ. Carolinae, 48 (2007), 607-622.

[10] F. Benamor and K. Boulabiar, Maximal ideals of disjointness preserving operators, J. Math. Anal. Appl., 322 (2006), 599-609.

[11] F. Benamor and K. Boulabiar, On the modulus of disjointness preserving operators on complex vector lattices, Algebra Univ., 54 (2005), 185-193.

[12] S. J. Bernau, Orthomorphisms of Archimedean vector lattices, Math. Proc. Cambridge Philos. Soc., 89 (1981), 119-128.

[13] S. J. Bernau, C. B. Huijsmans, and B. de Pagter, Sums of lattice homomorphisms, Proc. Amer. Math. Soc., 115 (1992), 151-156.

[14] A. Bigard and K. Keimel, Sur les endomorphismes conservant les polaires d'un groupe réticulé archimédien, Bull. Soc. Math. France, 97 (1969), 381398.

[15] G. Birkhoff and R. S. Pierce, Lattice-ordered rings, An. Acad. Brasil. Ciènc., 28 (1956), 41-69.

[16] K. Boulabiar, Order bounded separating linear maps on $\Phi$-algebras, Houston J. Math., 30 (2004), 1143-1155.

[17] K. Boulabiar and G. Buskes, A note on bijective disjointness preserving operators, in: Positivity IV - Theory and Applications, pp. 29-33, Technische Universität Dresden, 2006.

[18] K. Boulabiar and G. Buskes, After the determinants are down: a criterion for invertibility, Amer. Math. Monthly, 119 (2003), 737-741.

[19] K. Boulabiar and G. Buskes, Polar decomposition of order bounded disjointness preserving operators, Proc. Amer. Math. Soc., 132 (2004), 799-806.

[20] K. Boulabiar, G. Buskes, and M. Henriksen, A Generalization of a Theorem on Biseparating Maps, J. Math. Ana. Appl., 280 (2003), 334-339.

[21] K. Boulabiar, G. Buskes, and G. Sirotkin, Algebraic order bounded disjointness preserving operators and strongly diagonal operators, Integral Equa. Opera. Theory, 54 (2006), 9-31.

[22] K. Boulabiar, G. Buskes, and A. Triki, Recent trends and advances in certain lattice-ordered algebras, Contemporary Math., 328 (2003), 99-133.

[23] K. Boulabiar, G. Buskes, and A. Triki, Results in $f$-algebras, in: Positivity (Boulabiar et al. Eds), Trends in Mathematics, pp 73-96, Birkhäuser, BaselBoston-Berlin, 2007.

[24] P. F. Conrad and J. E. Diem, The ring of polar preserving endomorphisms on an Abelian lattice-ordered group, Illinois J. Math., 15 (1971), 222-240.

[25] E. Čech, On bicompact spaces, Ann. Math., 38 (1937), 823-844.

[26] Z. Ercan and S. Önall, A remark on the homomorphism on $C(X)$, Proc. Amer. Math. Soc., 133 (2005), 3609-3611.

[27] L. Gillman and M. Jerison, Rings of Continuous Functions, Springer Verlag, Berlin-Heidelberg-New York, 1976. 
[28] J. J. Grobler and C. B. Huijsmans, Disjointness preserving operators on complex Riesz spaces, Positivity, 1 (1997), 155-164.

[29] A. W. Hager, Isomorphism with a $C(Y)$ of the maximal ring of quotients of $C(X)$, Fund. Math., 66 (1969), 7-13.

[30] D. R. Hart, Some properties of disjointness preserving operators, Indag. Math., 88 (1985), 183-197.

[31] M. Henriksen, On the equivalence on the ring, lattice, and semigroup of continuous functions, Proc. Amer. Math. Soc., 7 (1956), 959-960.

[32] E. Hewitt, Rings of real-valued continuous functions I, Trans. Amer. Math. Soc., 64 (1948), 54-99.

[33] C. B. Huijsmans and B. de Pagter, Invertible disjointness preserving operators, Proc. Edinburgh Math. Soc., 37 (1993), 125-132.

[34] C. B. Huijsmans and A. W. Wickstead, The inverse of band preserving and disjointness preserving operators, Indag. Math., 3 (1992), 179-183.

[35] K. Jarosz, Automatic continuity of separating linear isomorphisms, Bull. Canadian Math. Soc. 33 (1990), 139-144.

[36] J. S. Jeang and N. C. Wong, Weighted composition operators of $C_{0}(X)$ 's, J. Math. Anal. Appl., 201 (1996), 981-993.

[37] I. Kaplansky, Infinite Abelian Groups, University of Michigan Press, Ann Arbor, 1954.

[38] Y. I. Karlovich and V. T. Kravchenko, Singular integral equations with non Carleman shift on anopen contour, Diff. Equat., 17 (1981), 2212-2223.

[39] S. S. Kukatuladze, Support set for sublinear operators, Soviet Math. Dokl., 21 (1976), 1428-1431.

[40] W. A. J. Luxemburg and A. R. Schep, A Radon-Nikodym type theorem for positive operators and a dual, Indag. Math., 40 (1978), 357-375.

[41] W. A. J. Luxemburg and A. C. Zaanen, Riesz spaces I, North-Holland, Amsterdam-London, 1971.

[42] M. Meyer, Les homomorphismes d'espaces vectoriels réticulés complexes, $C$. R. Acad. Sci. Paris Serie I, 292 (1981), 793-796.

[43] M. Meyer, Le stabilateur d'un espace vectoriel réticulé, C.R. Acad. Sci. Paris, Serie I, 283, (1976), 249-250.

[44] M. Meyer, Quelques propriétés des homomorphismes d'espaces vectoriels réticulés, Equipe d'Analyse Paris VI, preprint 131, 1979.

[45] P. Meyer-Nieberg, Banach Lattices, Springer, Berlin-Heidelberg-New York, 1991.

[46] A. D. Myshkis, Certain problems of the theory of differential equations with deviation argument, Uspechi Mat. Nauk., 32 (1974), 173-202.

[47] B. de Pagter, A note on disjointness preserving operators, Proc. Amer. Math. Soc., 90 (1984), 543-549.

[48] B. de Pagter, $f$-Algebras and Orthomorphisms, Thesis, Leiden, 1981.

[49] B. de Pagter, The space of extended orthomorphisms on a vector lattice, Pacific J. Math., 112 (1984), 193-210.

[50] B. de Pagter and A. R. Schep, Band decomposition for disjointness preserving operators, Positivity, 4 (2000), 259-288.

[51] R. J. Sakel and C. R. Sell, A spectral theory for linear differential systems, J. Diff. Equat., 27 (1978), 320-328. 
[52] T. Shirota, A class of topplogical spaces, Osaka Math. J., 4 (1952), 23-40.

[53] M. H. Stone, Applications of the theory of Boolean rings to general topology, Trans. Amer. Math. Soc., 41 (1937), 275-481.

[54] A. Tychonoff, Über die topologische Erweiterung von Raümen, Math. Ann., 102 (1930), 544-561.

[55] B. Z. Vulikh, On linear multiplicative operations, Dokl. Akad. Nauk., 41 (1943), 148-151.

[56] A. W. Wickstead, Representation and duality of multiplication operators on Archimedean Riesz spaces, Compositio Math., 35 (1977), 225-238.

[57] A. W. Wickstead, The injective hull of an Archimedean $f$-algebra, Compositio Math., 62 (1987), 329-342.

[58] A.C. Zaanen, Examples of orthomorphisms, J. Approx. Theory, 13 (1975) 192-204.

[59] A. C. Zaanen, Introduction to Operator Theory in Riesz Spaces, Springer Verlag, Berlin-Heidelberg-New York, 1997.

[60] A. C. Zaanen, Riesz Spaces II, North-Holland, Amsterdam-London, 1983.

Karim Boulabiar,

Institut Préparatoire aux Etudes Scientifiques et Techniques,

Université du 7 Novembre à Carthage,

BP 51, 2070-La Marsa, Tunisia

karim.boulabiar@ipest.rnu.tn

Received on 22 January 2008 and in revised form on 24 December 2008. 